\title{
Screening for Glaucomatous Visual Field Defects in Rural Australia with an iPad
}

\author{
Mark A Chia ${ }^{1}$, Edward Trang ${ }^{2}$, Ashish Agar ${ }^{3}$, Algis J Vingrys ${ }^{4}$, Jenny Hepschke ${ }^{5}$, George YX Kong ${ }^{6}$, Angus W Turner ${ }^{7}$
}

\begin{abstract}
Aim and objective: Developing improved methods for early detection of visual field defects is pivotal to reducing glaucoma-related vision loss. The Melbourne Rapid Fields screening module (MRF-S) is an iPad-based test, which allows suprathreshold screening with zone-based analysis to rapidly assess the risk of manifest glaucoma. The versatility of MRF-S has potential utility in rural areas and during infectious pandemics. This study evaluates the utility of MRF-S for detecting field defects in non-metropolitan settings.

Materials and methods: This was a prospective, multicenter, cross-sectional validation study. Two hundred and fifty-two eyes of 142 participants were recruited from rural sites through two outreach eye services in Australia. Participants were tested using MRF-S and compared with a reference standard; either Zeiss Humphrey Field Analyzer or Haag-Streit Octopus performed at the same visit. Standardized questionnaires were used to assess user acceptability. Major outcome measures were the area under the curve (AUC) for detecting mild and moderate field defects defined by the reference tests, along with corresponding performance characteristics (sensitivity, specificity).

Results: The mean test duration for MRF-S was 1.88 minutes compared with 5.92 minutes for reference tests. The AUCs for mild and moderate field defects were 0.81 [95\% confidence interval ( $\mathrm{Cl}$ ): $0.75-0.87$ ] and 0.87 ( $95 \% \mathrm{Cl}$ : 0.83-0.92), respectively, indicating very good diagnostic accuracy. Using a risk criterion of 55\%, MRF-S identified moderate field defects with a sensitivity and specificity of 88.4 and $81.0 \%$, respectively. Conclusion and clinical significance: The MRF-S iPad module can identify patients with mild and moderate field defects while delivering favorable user acceptability and short test duration. This has potential application within rural locations and amidst infectious pandemics.

Keywords: Computers, Cross-Sectional study, Glaucoma, Handheld, Mass screening, Visual field tests, Visual fields.

Journal of Current Glaucoma Practice (2021): 10.5005/jp-journals-10078-1312
\end{abstract}

\section{INTRODUCTION}

Glaucoma is a group of optic neuropathies characterized by progressive degeneration of retinal ganglion cells and irreversible visual field loss. ${ }^{1}$ It affects 70 million people worldwide and causes bilateral blindness in $10 \%$, making it the leading cause of irreversible blindness. ${ }^{1}$ Since the condition typically remains asymptomatic until more advanced stages of the disease, more than half of patients with glaucoma are unaware of their disease. ${ }^{2}$ Given there is substantial evidence from randomized trials that lowering intraocular pressure (IOP) reduces progression, ${ }^{3,4}$ developing new methods for early diagnosis of glaucoma in a community setting is critically important. The challenge of timely diagnosis of glaucoma is even greater within rural areas, where there is limited access to standard ophthalmic care.

Currently, there are no practical and cost-effective population screening tests for detecting visual field defects. ${ }^{5}$ Early glaucoma screening efforts have focused on IOP assessment; however, these types of classifications lead to unacceptably high rates of false-negatives. 5,6 Open-angle glaucoma may be associated with a normal IOP in $>35 \%$ of cases. ${ }^{5}$ Other screening methods which involve objective observation or imaging of the optic disk have similarly failed to improve on this poor sensitivity. ${ }^{6}$ More recently, evaluation of the peripheral visual fields has been explored as a more reliable approach to glaucoma screening. ${ }^{7,8} \mathrm{~A}$ communitybased study involving 4,744 participants, from urban and rural communities, found that the presence of visual field loss was the most substantial and significant variable for detecting cases of undiagnosed glaucoma. ${ }^{9}$

Traditional perimetry remains an essential part of documenting the presence and severity of glaucoma through well-described $\overline{\text { 1,7 Lions Outback Vision, Lions Eye Institute, Nedlands, Western }}$ Australia, Australia; Centre for Ophthalmology and Visual Science, University of Western Australia, Nedlands, Western Australia, Australia

${ }^{2}$ Faculty of Medicine, University of New South Wales, Kensington, New South Wales, Australia

3,5 Faculty of Medicine, University of New South Wales, Kensington, New South Wales, Australia; Department of Ophthalmology, Prince of Wales Hospital, Randwick, New South Wales, Australia

${ }^{4}$ Department of Optometry and Vision Sciences, University of Melbourne, Parkville, Victoria, Australia

${ }^{6}$ Department of Glaucoma, Royal Victorian Eye and Ear Hospital, East Melbourne, Victoria, Australia; Centre for Eye Research Australia, East Melbourne, Victoria, Australia

Corresponding Author: Mark A Chia, Lions Outback Vision, Lions Eye Institute, Nedlands, Western Australia, Australia; Centre for Ophthalmology and Visual Science, University of Western Australia, Nedlands, Western Australia, Australia, Phone: +61 893810802, e-mail: mark.a.chia@outlook.com

How to cite this article: Chia MA, Trang E, Agar A, et al. Screening for Glaucomatous Visual Field Defects in Rural Australia with an iPad. J Curr Glaucoma Pract 2021;15(3):125-131.

Source of support: George Kong received grant funding from the Ophthalmic Research Institute of Australia, Royal Victorian Eye and Ear Hospital (East Melbourne VIC 3002), and Glaucoma Australia (Artarmon NSW 2064)

Conflict of interest: George Kong and Algis Vingrys are Founding Directors of Glance Optical Pty. Ltd., the maker of the Melbourne Rapid Fields software

(O) The Author(s). 2021 Open Access This article is distributed under the terms of the Creative Commons Attribution 4.0 International License (https://creativecommons. org/licenses/by-nc/4.0/), which permits unrestricted use, distribution, and non-commercial reproduction in any medium, provided you give appropriate credit to the original author(s) and the source, provide a link to the Creative Commons license, and indicate if changes were made. The Creative Commons Public Domain Dedication waiver (http://creativecommons.org/publicdomain/zero/1.0/) applies to the data made available in this article, unless otherwise stated. 
patterns of visual field loss. ${ }^{10}$ Despite this, there are significant shortcomings that limit the use of perimetry as a screening tool. ${ }^{8}$ The most commonly used perimeters cost above USD 20,000 and weigh around $30 \mathrm{~kg}$. The typical test duration is approximately 6 minutes per eye $\mathrm{e}^{11}$ and requires a high level of concentration by the patient. Tests commonly need to be repeated on several occasions to produce consistent results. ${ }^{12}$ Specialist equipment of this nature can be difficult to finance within rural locations where patient numbers may not justify their expense, and lack of portability can be a significant barrier to their use by visiting outreach specialist services.

Since the advent of the 2019 coronavirus disease (COVID-19), new challenges have arisen relevant to the administration of visual field tests. Following the outbreak of the COVID-19 pandemic, several ophthalmological representative bodies issued statements urging eye services to immediately cease any treatment other than urgent or emergent care. ${ }^{13,14}$ This is problematic as traditional field testing cannot be performed remotely. Guidelines from the Royal Australian and New Zealand College of Ophthalmologists recommend considering whether close-contact tests such as visual fields are necessary and minimizing these where possible. ${ }^{14}$

Due to the direct patient contact involved, any ongoing testing requires time-consuming disinfection procedures to be performed between individual patients, which has resulted in many services ceasing field testing altogether. American Academy of Ophthalmology guidelines highlight the importance of following manufacturer's instructions for disinfection between patients. ${ }^{13}$ Instructions for the Humphrey Field Analyzer (HFA) warn against the risk of damage to the bowl while using a rubbing motion against the surface and instead recommends the use of an atomizing sprayer followed by leaving to dry for a full 10-15 minutes. ${ }^{15}$ Guidelines for the Octopus by Haag-Streit caution that even using an atomizer spray can cause damage to the surface with frequent use. ${ }^{16}$

Recent advances in modern portable tablet devices such as the Apple iPad have resulted in high-resolution displays that can be utilized for visual field testing in a more versatile way. Melbourne Rapid Fields (MRF) is an iPad application, which was designed as an inexpensive and portable method of assessing visual fields. ${ }^{17}$ As portable tablets become more ubiquitous in the general population, a significant advantage is their potential utility outside traditional clinical environments, such as within rural areas or for home-monitoring during viral pandemics. Disinfection procedures for an iPad are straightforward as surfaces may be wiped regularly without causing damage.

The MRF application allows assessment in two modes: a full threshold test of 4-5 minutes duration per eye and a screening test of approximately 90 seconds duration. The full-threshold module has been evaluated in several studies..$^{11,17-19}$ Kong et al. ${ }^{19}$ compared global indices between MRF and HFA in a cohort of 90 participants from a glaucoma clinic. They found high concordance between mean deviation (MD) and pattern deviation, and similar test-retest reliability. Schulz et al. ${ }^{18}$ performed the first independent evaluation of MRF and found good performance characteristics compared with HFA for detection of defects, correlation of global indices, and regional mean threshold values. The Melbourne Rapid Fields screening module (MRF-S) has not previously been validated.

Visual fields easy (VFE) is a prototype suprathreshold iPad application, which has since been replaced by MRF-S following improvements to the underlying test logic. A previous study showed that VFE was able to detect moderate and severe visual field defects; however, it performed poorly for patients with mild defects and suffered from a high false-positive rate. ${ }^{20}$ The test specifications for VFE have previously been described elsewhere. ${ }^{20}$

The rapid screening module MRF-S was derived from the fullthreshold MRF test using intensity just above threshold (minimum required suprathreshold) at each test location. This was chosen to ensure that stimuli had high specificity and sensitivity to detect subtle field defects. Improving on the VFE application, the algorithm contains test logic that retests abnormal locations up to three times to reduce false-positive results. The module also performs risk calculations based on zone analysis of field defects to increase the specificity of results. It is intended for use as a rapid screening tool to detect visual field defects, particularly in areas where a standard perimeter would not be practical due to cost and transportation logistics.

Previous studies have not evaluated MRF outside of a metropolitan clinic environment or formally assessed user acceptability. Several modifications have also been made to the practical aspects of the testing procedure, including the addition of a Bluetooth keyboard for improved tactile feedback and the use of a customized hood to fix the viewing distance and prevent light interference from external sources. The primary aim of this study was to assess the ability of MRF-S to detect visual field defects in patients from rural and remote settings, with a secondary aim of evaluating user acceptability across several domains.

\section{Materials and Methods Study Design}

This was a prospective, multicenter, cross-sectional validation study. Ethics approval was obtained from the University of Western Australia Human Research Ethics Committee (RA/4/20/4146). The study was performed in accordance with the tenets of the Declaration of Helsinki. All participants gave informed consent before inclusion.

\section{Participants}

To consider the general applicability of the MRF test, participants were recruited from several rural sites in Australia through two separate clinical services: Lions Outback Vision (LOV) and Outback Eye Service (OES). Lions Outback Vision is a visiting mobile ophthalmology clinic, which services rural towns across the state of Western Australia. Outback Eye Service is the outreach service from the Prince of Wales Hospital in Sydney. It provides the sole public ophthalmology service for western New South Wales (NSW), serving regional and remote centers across two-thirds of the state. Referrals to both services are sourced from a combination of general medical practitioners and optometrists.

Inclusion criteria were: (1) age over 50 years or 40 years with a family history of glaucoma, (2) best-corrected visual acuity of at least $6 / 12$ in the included eye, and (3) no history of intraocular surgery within the last 3 months. There were no additional exclusion criteria based on the presence of comorbidities such as diabetes or hypertension. Participants consisted of a convenience sample recruited between February and October 2019. Both eyes were included unless one of the eyes failed to meet inclusion criteria as specified above. While participants had not previously performed MRF testing, most had experience with other forms of visual field exam.

\section{Test Procedure}

Participants underwent routine clinical examination and investigations including IOP measurement, slit-lamp examination, 
and ocular coherence tomography of the optic disk (Topcon 3D OCT-1 Maestro). Participants then proceeded to visual field testing with MRF-S and a reference test (see later) on the same day in random order. Following testing, the glaucoma status of each patient was recorded as determined by a trained ophthalmologist.

Melbourne Rapid Fields screening module testing was performed in a dark room free from distractions with appropriate near correction and occlusion of the fellow eye. An iPad Pro Generation 1 (Apple, Cupertino, CA, USA) connected to a Bluetooth keyboard running the MRF-S application was prepared within a custom viewing hood to shield unwanted light from the screen and fix the viewing distance at $33 \mathrm{~cm}$. Participants used the keyboard spacebar as a response button to indicate when a stimulus had been seen. Standardized instructions were provided, and a brief practice trial was performed before commencing the test. If both eyes met inclusion criteria they were tested consecutively. Figure 1 demonstrates the patient setup during testing with an illustrative sample of the screen and the result from the MRF-S application.

The reference test was chosen based on the availability of equipment at the recruitment site. Participants recruited through LOV were tested with the Octopus 600 24-2 dynamic strategy (HaagStreit, Koniz, Switzerland) while patients recruited through OES used the Humphrey Field Analyzer 2 24-2 SITA Standard strategy (Carl Zeiss, Oberkochen, Germany) both of which have been extensively validated. ${ }^{21-23}$ Reference field tests were conducted in a dark room according to the manufacturer's instruction with the appropriate trial lens.

A subset of participants was chosen at random to complete a short iPad-based questionnaire designed to evaluate user acceptability. Participants were asked to rate the simplicity, comfort, and overall test experience on a Likert scale between 1 and 5 for both MRF-S and the reference test. Test durations were recorded for each visual field test.

\section{Statistical Analysis}

We calculated the minimum sample size required for validation of this screening test using a method described by Bujang and Adnan. ${ }^{24}$ Assuming a field defect prevalence of $20 \%$, we required sample size of 245 participants (including 49 with visual field defects) to achieve a power of $80 \%$ for detecting a change in the percentage value of sensitivity from 0.50 to 0.70 , based on a target significance level of $0.05 .^{24}$

We used two different MD cut-offs to define a positive reference test. An MD less than -6 was used to define moderate field defects and worse, and an MD less than -3 defined mild field defects and worse. Cut-offs were chosen based on previously described definitions, ${ }^{25}$ with consideration for the severity of defect that is clinically important to detect in a screening population while balancing the issue of high false-positives. The summary measure provided by MRF-S is a percentage risk of abnormality ranging from 2-99\% (MRF-S Risk). This score is calculated with an algorithm based on a number of missed points, giving greater weight to missed points that form a cluster within the same zone. We did not prespecify test positivity cut-offs for MRF-S Risk but planned to identify cut-offs based on exploratory analyzes of receiver operating characteristics (ROC) curves derived using the MRF-S risk index. Although glaucoma status was recorded to describe the study population, we did not use glaucoma status to define cases positive for a field defect. Reference positive cases were defined solely according to MD scores returned from the Octopus or HFA tests.

We classified a test with poor reliability as having $>33 \%$ falsepositives. Unreliable tests on either MRF-S or the reference test were excluded from the analysis. We did not use false-negatives to define reliability due to biological explanations for high falsenegatives in those with known visual field defects. ${ }^{26,27}$ Melbourne

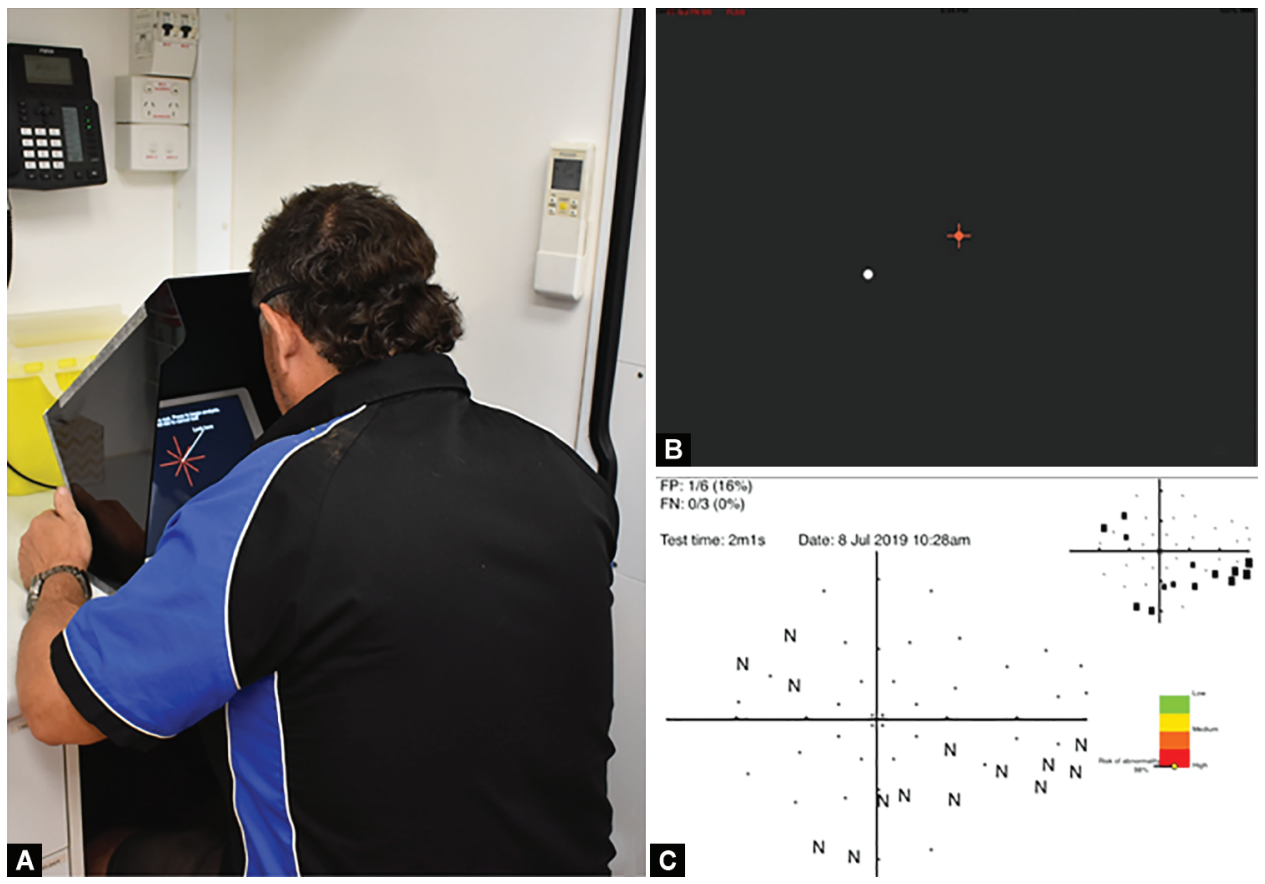

Figs $1 \mathrm{~A}$ to C: (A) Patient positioning and iPad setup during the testing procedure; (B) Screenshot during testing with the red fixation cross and a test spot shown at lower left; $(C)$ Sample output of Melbourne Rapid Fields showing the inferior nasal defect. MRF-S Risk output is shown in the lower right 
Rapid Fields screening module does not record fixation losses but provides voice prompts to maintain fixation.

Statistical analysis was performed using SPSS Statistics 26 (IBM, New York, USA). Melbourne Rapid Fields screening module Risk results were used to produce ROC curves for both moderate (MD worse than -6) and mild (MD worse than -3) visual field defects and the area under the curve (AUC) was calculated. Receiver operating characteristics analysis is a widely used method of evaluating diagnostic accuracy in which the performance indices are not impacted by arbitrarily defined thresholds. ${ }^{28}$ By exploring the ROC curves, we chose an appropriate MRF-S Risk cut-off and utilized this to calculate performance characteristics (sensitivity, specificity, positive and negative predictor values, positive and negative likelihood ratios). Correlation coefficients were used to describe the relationship between MRF-S Risk and MD. To evaluate user acceptability, we compared mean Likert scores and test durations using paired sample $t$-tests, with a $p$ value of $<0.05$ to indicate significance.

\section{Results}

\section{Participants}

Our study included 252 eyes of 142 participants. Of these, 111 eyes of 57 patients were recruited from LOV and 141 eyes of 85 patients were recruited from OES. Three eyes were excluded from the statistical analysis because of poor reliability due to high falsepositives, with 249 eyes remaining in the final analysis. All three of the unreliable tests were performed on the MRF-S test. A patient flow diagram is presented in Flowchart 1.

Baseline demographic and clinical characteristics of participants are presented in Table 1 according to their recruitment site. The mean age of participants was 63.4 years at LOV and 70.9 years at OES. The LOV cohort $(n=57)$ consisted of 23 glaucoma patients, 18 glaucoma suspects, and 16 patients without signs of glaucoma, using definitions outlined by Foster et al. ${ }^{29}$ The OES cohort $(n=85)$ consisted of 67 patients with glaucoma and 18 glaucoma suspects. Mean IOP was $17.5 \mathrm{~mm} \mathrm{Hg}$ among the LOV cohort and $14.4 \mathrm{~mm}$ $\mathrm{Hg}$ among the OES cohort. Patients used glaucoma treatment as deemed appropriate by their usual practitioner.

\section{Diagnostic Accuracy}

Receiver operating characteristics curves illustrating the diagnostic ability of MRF-S to identify mild and moderate visual fields defects at various MRF-S risk levels are presented in Figure 2. Selected coordinates of the ROC curves showing the corresponding sensitivities and specificities are presented in Table 2. The AUCs for mild and moderate visual field defects were 0.81 [95\% confidence interval $(\mathrm{Cl})$ 0.75-0.87] and 0.87 (95\% Cl 0.83-0.92), respectively, indicating "very good" diagnostic accuracy according to the classification by Šimundić. ${ }^{30}$

By examining the ROC curve and corresponding sensitivities and specificities, an MRF-S Risk of $\geq 55 \%$ was chosen for identifying moderate field defects (gray in Table 2). Similarly, an MRF-S Risk criterion of $25 \%$ was chosen for identifying mild field defects (gray in Table 2). Cross-tabulation of the MRF-S results against the reference field test is presented in Table 3. Table 4 presents various measures of diagnostic accuracy using the selected MRF-S Risk criteria. Pearson's correlation coefficient between MRF-S Risk and reference MD scores was $-0.664(95 \% \mathrm{Cl}-0.758$ to -0.571$)$, indicating a moderately strong negative correlation (higher MRF-S risk correlates with more negative MD). ${ }^{31}$

Table 1: Demographic and clinical characteristics of participants

\begin{tabular}{lll}
\hline Characteristic & $\begin{array}{l}\text { Lions Outback Vi- } \\
\text { sion } n=57\end{array}$ & $\begin{array}{l}\text { Outback Eye Service } \\
n=85\end{array}$ \\
\hline $\begin{array}{l}\text { Age (years), mean (SD) } \\
\text { Sex, } n \text { (\%) }\end{array}$ & $63.4(9.5)$ & $70.9(15.7)$ \\
$\quad$ Male & $35(61)$ & $52(61)$ \\
$\quad$ Female & $22(39)$ & $33(39)$ \\
BCVA (LogMAR), mean & $0.10(0.11)$ & $0.14(0.14)$ \\
(SD) & & $14.4(3.9)$ \\
IOP (mm Hg), mean (SD) & $17.5(5.2)$ & \\
Glaucoma status, $n$ (\%) & & $67(79)$ \\
$\quad$ Glaucoma & $23(40)$ & $18(21)$ \\
$\quad$ Glaucoma suspect & $18(32)$ & \\
$\quad$ Normal & $16(28)$ &
\end{tabular}

SD, standard deviation; BCVA, best-corrected visual acuity; IOP, intraocular pressure

Flowchart 1: Flow diagram of participant inclusion

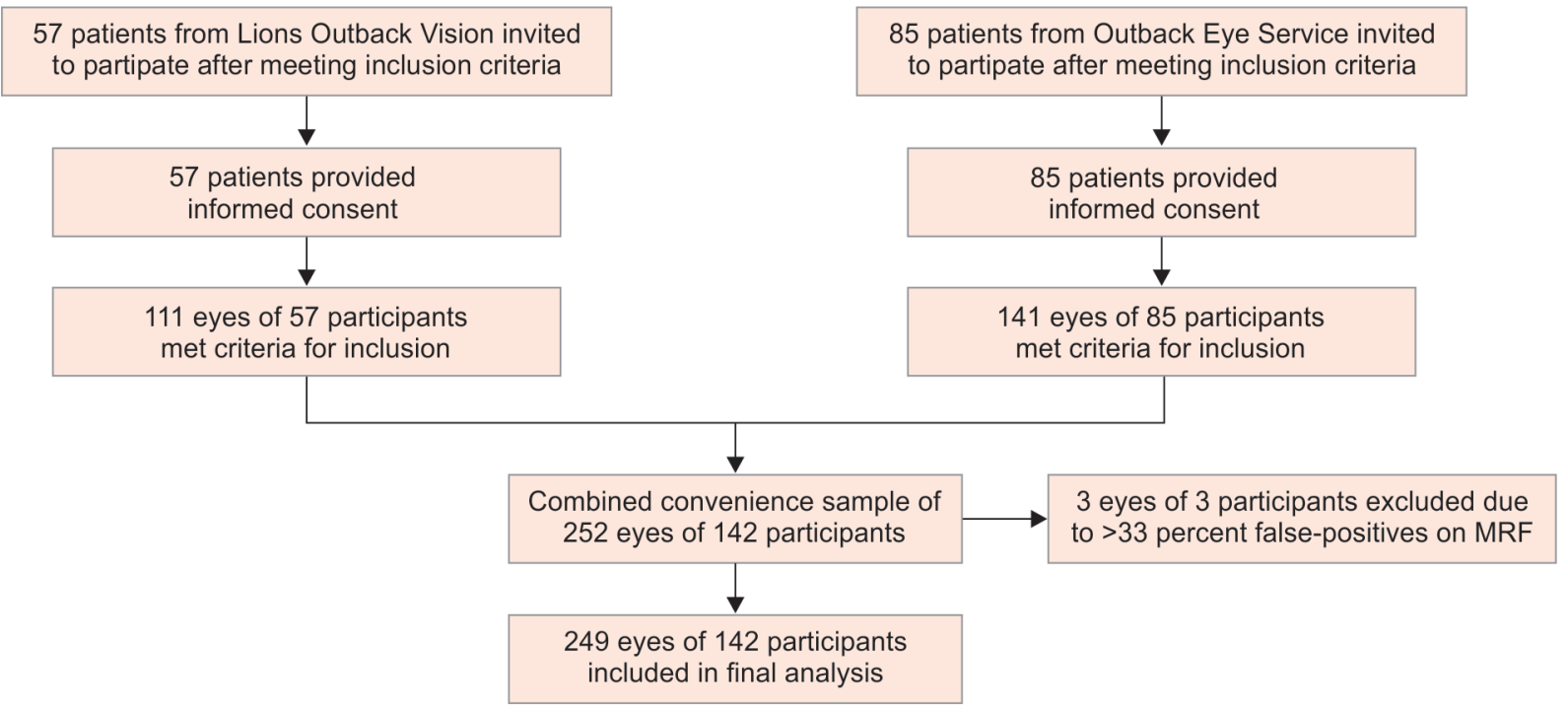



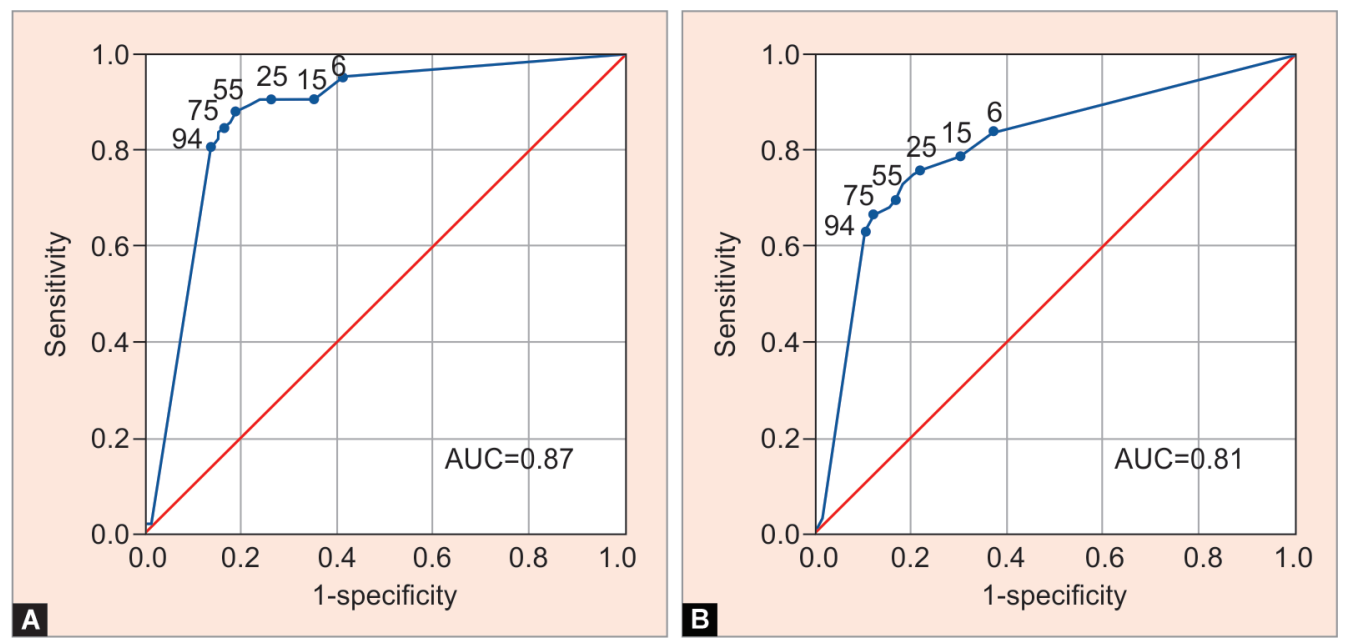

Figs $2 \mathrm{~A}$ and B: Receiver operating characteristic curves for detecting moderate (mean deviation $\leq-6 \mathrm{~dB}$, $(\mathrm{A})$ and mild (mean deviation $\leq-3 \mathrm{~dB}$, (B) defects on the Octopus or Humphrey Field Analyzer for different levels of risk returned from the Melbourne Rapid Fields-Screening test

Table 2: Sensitivity and specificity of Melbourne Rapid Fields-Screening test for various MRF-S risk levels compared with mean deviation returned from reference test

\begin{tabular}{lllll}
\hline & \multicolumn{2}{c}{$\begin{array}{c}\text { Moderate field defect } \\
(M D \leq-6 d B)\end{array}$} & \multicolumn{2}{c}{$\begin{array}{c}\text { Mild field defect } \\
(M D \leq-3 d B)\end{array}$} \\
\cline { 2 - 5 } $\begin{array}{l}\text { MRF-S } \\
\text { Risk } \\
\text { level (\%) }\end{array}$ & $\begin{array}{l}\text { Sensitivity } \\
\text { (\%) }\end{array}$ & Specificity (\%) & $\begin{array}{l}\text { Sensitivity } \\
(\%)\end{array}$ & $\begin{array}{l}\text { Specificity } \\
(\%)\end{array}$ \\
\hline 6 & 95.3 & 58.9 & 83.7 & 63.5 \\
15 & 90.7 & 65.0 & 78.9 & 69.8 \\
25 & 90.7 & 73.0 & 76.4 & 77.8 \\
35 & 90.7 & 76.1 & 74.8 & 80.2 \\
45 & 89.5 & 77.9 & 73.2 & 81.7 \\
55 & 88.4 & 81.0 & 69.9 & 83.3 \\
65 & 86.0 & 82.2 & 68.3 & 84.9 \\
75 & 83.7 & 84.7 & 66.7 & 88.1 \\
94 & 80.2 & 86.5 & 63.4 & 89.7 \\
98.5 & 4.7 & 98.2 & 4.1 & 98.4 \\
\hline
\end{tabular}

MRF-S, Melbourne Rapid Fields-Screening; MD, mean deviation

\section{User Acceptability}

A random subset of 117 participants ( $\mathrm{LOV}=32 ; \mathrm{OES}=85$ ) completed a survey to assess the simplicity, comfort, and overall test experience of MRF-S compared with the reference visual field tests (Octopus or HFA). Participants rated the simplicity of MRF-S significantly higher $(t=9.59, \mathrm{df}=115, p<0.001)$ with a mean Likert scale score of 4.61 $(\mathrm{SD}=0.69)$ compared with $3.47(\mathrm{SD}=1.22)$. Melbourne Rapid Fields screening module was also rated as more comfortable ( $t=3.07$, df $=30, p=0.002)$, with a mean score of $4.26(\mathrm{SD}=0.86)$ compared with 3.47 ( $S D=1.22)$. Overall test experience was higher for MRF-S $(t=9.20, \mathrm{df}=116, p<0.001)$, with a mean score of $4.41(\mathrm{SD}=0.87)$ compared with 3.23 (SD = 1.32).

Melbourne Rapid Fields screening module took an average of 1 minute 53 seconds to complete across all participants compared to 5 minutes 55 seconds for the reference tests. Melbourne Rapid Fields screening module had a shorter duration by 4.03 minutes (95\% Cl 3.82-4.24) compared with the reference tests $(t=37.3$, df $=229, p<0.001)$. There were no adverse events identified from performing either MRF-S or the reference visual field tests.
Table 3: Cross-tabulation of Melbourne Rapid Fields-Screening risk against reference test mean deviation

\begin{tabular}{|c|c|c|c|}
\hline \multicolumn{4}{|c|}{ Moderate field defect } \\
\hline & $\begin{array}{l}\text { Reference positive } \\
(M D \leq-6)\end{array}$ & $\begin{array}{l}\text { Reference nega- } \\
\text { tive }(M D>-6)\end{array}$ & Total \\
\hline $\begin{array}{l}\text { MRF-S positive } \\
\text { (Risk } \geq 55 \%)\end{array}$ & 76 & 31 & 107 \\
\hline $\begin{array}{l}\text { MRF-S negative } \\
\text { (Risk <55\%) }\end{array}$ & 10 & 132 & 142 \\
\hline Total & 86 & 163 & 249 \\
\hline \multicolumn{4}{|c|}{ Mild field defects } \\
\hline & $\begin{array}{l}\text { Reference positive } \\
(M D \leq-3)\end{array}$ & $\begin{array}{l}\text { Reference nega- } \\
\text { tive }(M D>-3)\end{array}$ & Total \\
\hline $\begin{array}{l}\text { MRF-S positive } \\
\text { (Risk } \geq 25 \%)\end{array}$ & 94 & 28 & 122 \\
\hline $\begin{array}{l}\text { MRF-S negative } \\
\text { (Risk }<25 \% \text { ) }\end{array}$ & 29 & 98 & 127 \\
\hline Total & 123 & 126 & 249 \\
\hline
\end{tabular}

MRF-S, Melbourne Rapid Fields-Screening; MD, mean deviation

\section{Discussion}

Our study demonstrates that MRF-S can identify moderate visual field defects when compared with traditional field machines with very good diagnostic accuracy. Using an MRF-S risk criterion of $55 \%$, MRF-S identified participants with moderate field defects with a sensitivity and specificity of 88.4 and $81.0 \%$, respectively. Melbourne Rapid Fields screening module can also identify mild field defects with very good diagnostic accuracy. Adopting an MRF-S risk criterion of $25 \%$, MRF-S identified mild field defects with a sensitivity and specificity of 76.4 and $77.8 \%$, respectively. Based on the results of a standardized questionnaire, MRF-S was rated as being simpler and more comfortable for participants than traditional field testing, with a superior overall test experience. Test durations were on average 4 minutes shorter compared with the reference field tests.

The results of our study are comparable or superior to previously published studies evaluating the utility of screening tests 
Table 4: Diagnostic accuracy measures with $95 \%$ confidence intervals Moderate field defect with 55\% MRF-S risk criterion

\begin{tabular}{|c|c|c|c|}
\hline & Estimate & Lower limit & Upper limit \\
\hline Sensitivity (\%) & 88.4 & 79.2 & 94.0 \\
\hline Specificity (\%) & 81.0 & 74.0 & 86.5 \\
\hline Positive predictive value (\%) & 71.0 & 61.3 & 79.2 \\
\hline Negative predictive value (\%) & 92.9 & 87.1 & 96.4 \\
\hline Positive likelihood ratio & 4.65 & 3.35 & 6.44 \\
\hline Negative likelihood ratio & 0.14 & 0.08 & 0.26 \\
\hline
\end{tabular}

Mild field defect with $25 \%$ MRF-S risk criterion

\begin{tabular}{lcll}
\hline & Estimate & Lower limit & Upper limit \\
\hline Sensitivity (\%) & 76.4 & 67.8 & 83.4 \\
Specificity (\%) & 77.8 & 69.3 & 84.5 \\
Positive predictive value (\%) & 77.0 & 68.4 & 84.0 \\
Negative predictive value (\%) & 77.2 & 68.7 & 83.9 \\
Positive likelihood ratio & 3.44 & 2.45 & 4.84 \\
Negative likelihood ratio & 0.30 & 0.22 & 0.42 \\
\hline
\end{tabular}

MRF-S, Melbourne Rapid Fields-Screening

for visual field defects. Tsapakis et al. ${ }^{8}$ performed a validation study on 20 eyes of 10 patients, comparing a computer-based field test with a Humphrey perimeter using three threshold levels: $-12 \mathrm{~dB}$ (low), $-8 \mathrm{~dB}$ (medium), and $-4 \mathrm{~dB}$ (high). Reported AUCs for their low, medium, and high thresholds were $0.762,0.782$, and 0.837 , respectively. ${ }^{8}$ After applying optimal cut-off points, they reported sensitivities and specificities of 63.7 and $73.5 \%$ for high threshold (mild loss), 79.0 and $64.6 \%$ for medium threshold (moderate loss), and 94.2 and $49.7 \%$ for low threshold. ${ }^{8}$ The duration of the screening test was approximately 2-3 minutes.

Peristat is a web-based suprathreshold perimeter, which can be performed on any computer monitor of at least 17 inches. Lowry et al. ${ }^{32}$ evaluated Peristat against Humphrey visual fields among 63 glaucoma patients and 30 healthy controls. They reported AUCs ranging from 0.77 to 0.81 for mild disease (MD $>-6 \mathrm{~dB}$ on $\mathrm{HFA}$ ) and 0.85 to 0.87 for moderate disease (MD $<-6 \mathrm{~dB}$ on HFA) depending on the chosen contrast threshold. ${ }^{32}$ This appears to be a similar level of performance to that observed in our study although the duration of the test was longer than MRF-S at around 5 minutes.

Rarebit is a computer-based test that can be performed on a 15-inch monitor, but again with a longer test duration of about 6 minutes. ${ }^{33}$ Brusini et al. ${ }^{33}$ evaluated various algorithms for distinguishing between controls and glaucomatous eyes using Rarebit and reported AUCs ranging from 0.89 to 0.95 . Interestingly, the Pearson's correlation coefficient between HFA MD and the Rarebit summary measure was low at 0.38 among glaucoma patients. ${ }^{33}$ One of the relevant methodological differences was the exclusions of patients with ocular hypertension from this analysis, which may lead to spectrum bias and would be expected to produce more favorable performance characteristics. ${ }^{33,34}$

Frequency doubling technology (FDT) is another relatively portable method of visual field testing, but with a higher cost than an iPad-based test. Robin et al. ${ }^{35}$ evaluated various glaucoma screening strategies on 659 participants. For FDT alone, reported sensitivities were $79-84 \%$ with specificities of $55-66 \%$ depending on the magnitude of loss. ${ }^{35}$ Melbourne Rapid Fields screening module appears to return better diagnostic performance for its screening test for mild and moderate defects (Table 4).
Important strengths of our study include: (1) its prospective design (2) diverse sampling from a rural cohort, and (3) evaluation of user experience in addition to test performance. The prospective nature of the study allowed us to consistently apply standardized testing procedures and to collect pre-specified data from all participants. The chosen population enabled the evaluation of a diverse rural cohort from multiple sites and two separate clinical services, reflecting the heterogeneity and real-world challenges of screening for visual field defects. These characteristics are relevant as the major benefits of affordability, portability, and shorter test durations are of particular importance within rural and remote areas.

Our study has several limitations. Patients were enrolled as a convenience sample which has the potential to introduce selection bias. ${ }^{36}$ Due to the broad inclusion criteria, it was not feasible to recruit patients consecutively within the constraints of operating a busy outreach specialty clinic. Within the cohort, there was variation in the level of previous experience with performing visual field tests. Although all participants were unfamiliar with the MRF-S test, most glaucoma patients had performed the reference tests on one or more occasions.

As the first validation study of the rapid MRF-S module, performance compared with similar tests is promising for its potential use in glaucoma screening. Despite this, further improvements to the test strategy are necessary. Although we have demonstrated the ability of MRF-S to identify field defects among a clinic population with a high burden of disease, specificities around $80 \%$ remain problematic for screening tests. When applied to a cohort of mostly healthy patients, numerous false-positives would be expected, impacting the costeffectiveness of a screening program. Further work is required to apply MRF-S outside of a clinic environment to establish accurate false-positive rates in a healthy cohort. A hybrid strategy, in which individuals initially identified as abnormal undergo repeat testing or sequential testing with MRF-S or the full MRF module, has the potential to improve specificity to a range more suitable for glaucoma screening.

In the context of the COVID-19 pandemic, many communities around the world are in a state of isolation with limited access to ophthalmic professionals. An advantage of MRF is its potential to detect and monitor visual field defects remotely without placing staff and patients at risk. The MRF application was specifically designed for this purpose, with in-built instructional voice prompts and remote sharing capabilities. While our study shows the utility of MRF-S in a non-metropolitan environment, further work evaluating MRF-S in an unsupervised home environment is required.

\section{Conclusion and Clinical Significance}

Our study demonstrates the potential of MRF-S to accurately identify patients with both mild and moderate visual field defects in rural locations. The test delivers a favorable user experience, with higher patient ratings for simplicity, comfort, and overall experience when compared with traditional field machines. The duration of MRF-S was significantly shorter than reference tests, addressing one of the important practical challenges associated with incorporating visual field assessment into glaucoma screening. Further study is required to explore the utility and cost-effectiveness of MRF-S within a large-scale population screening program and to evaluate its reliability within unsupervised environments. 


\section{References}

1. Weinreb RN, Aung T, Medeiros FA. The pathophysiology and treatment of glaucoma. J Am Med Assoc 2014;311(18):1901. DOI: 10.1001/jama.2014.3192.

2. Weinreb RN, Leung CKS, Crowston JG, et al. Primary open-angle glaucoma. Nat Rev Dis Primers 2016;2(1):16067. DOI: 10.1038/ nrdp.2016.67.

3. Agis Investigators. The Advanced Glaucoma Intervention Study (AGIS): 7. The relationship between control of intraocular pressure and visual field deterioration. Am J Ophthalmol 2000;130(4):429-440. DOI: 10.1016/s0002-9394(00)00538-9.

4. Heijl A, Leske MC, Bengtsson B, et al. Reduction of intraocular pressure and glaucoma progression: results from the Early Manifest Glaucoma Trial. Arch Ophthalmol 2002;120(10):1268-1279. DOI: 10.1001/archopht.120.10.1268.

5. lanchulev T, Pham P, Makarov V, et al. Peristat: A computer-based perimetry self-testfor cost-effective population screening of glaucoma. Curr Eye Res 2005;30(1):1-6. DOI: 10.1080/02713680490522399.

6. Quigley HA. Identification of glaucoma-related visual field abnormality with the screening protocol of frequency doubling technology. Am J Ophthalmol 1998;125(6):819-829. DOI: 10.1016/ s0002-9394(98)00046-4.

7. Bruun-Jensen J. Visual field screening with a laptop computer system. J Am Optom Assoc 2011;82(9):519-527. DOI: 10.1016/j. optm.2010.09.016.

8. Tsapakis S, Papaconstantinou D, Diagourtas A, et al. Home-based visual field test for glaucoma screening comparison with humphrey perimeter. Clin Ophthalmol 2018;12:2597. DOI: 10.2147/OPTH. S187832.

9. Wong EY, Keeffe JE, Rait JL, et al. Detection of undiagnosed glaucoma by eye health professionals. Ophthalmology 2004;111(8):1508-1514. DOI: 10.1016/j.ophtha.2004.01.029.

10. Atalay E, Nongpiur ME, Yap SC, et al. Pattern of visual field loss in primary angle-closure glaucoma across different severity levels. Ophthalmology 2016;123(9):1957-1964. DOI: 10.1016/j. ophtha.2016.05.026.

11. Prea SM, Kong YXG, Mehta A, et al. Six-month longitudinal comparison of a portable tablet perimeter with the humphrey field analyzer. Am J Ophthalmol 2018;190:9-16. DOI: 10.1016/j.ajo.2018.03.009.

12. Broadway DC. Visual field testing for glaucoma-a practical guide. Community Eye Health 2012;25(79-80):66.

13. American Academy of Ophthalmology. Important coronavirus updates for ophthalmologists. Available at http://www.aao.org. Accessed May 2, 2020.

14. Royal Australian and New Zealand College of Ophthalmologis. Corona Virus (COVID-19) Guidelines. Available at http://www.ranzco.edu. Accessed May 28, 2020.

15. Carl Zeiss Meditec. Cleaning Guidance for the Humphrey Field Analyzer. Available at http://www.zeiss.com. Accessed May 28, 2020.

16. Haag-Steit Diagnostics. Cleaning and Disinfection of Octopus Perimeters. Available at http://www.haag-streit.com. Accessed May 28, 2020.

17. Vingrys AJ, Healey JK, Liew S, et al. Validation of a Tablet as a Tangent Perimeter. TransI Vis Sci Technol. 2016;5(4):3-3. DOI: 10.1167/tvst.5.4.3.

18. Schulz AM, Graham EC, You Y, et al. Performance of iPad-based threshold perimetry in glaucoma and controls. Clin Experiment Ophthalmol 2018;46(4):346-355. DOI: 10.1111/ceo.13082.
19. Kong YXG, He M, Crowston JG, et al. A comparison of perimetric results from a tablet perimeter and Humphrey field analyzer in glaucoma patients. TransI Vis Sci Technol 2016;5(6):2-2. DOI: 10.1167/ tvst.5.6.2.

20. Johnson CA, Thapa S, Kong YXG, et al. Performance of an iPad application to detect moderate and advanced visual field loss in Nepal. Am J Ophthalmol 2017;182:147-154. DOI: 10.1016/j. ajo.2017.08.007.

21. Maeda $H$, Nakaura $M$, Negi A. New perimetric threshold test algorithm with dynamic strategy and tendency oriented perimetry (TOP) in glaucomatous eyes. Eye 2000;14(5):747. DOI: 10.1038/eye.2000. 196.

22. Scherrer M, Fleischhauer J, Helbig H, et al. Comparison of tendencyoriented perimetry and dynamic strategy in Octopus perimetry as a screening tool in a clinical setting: a prospective study. Klin Monbl Augenheilkd 2007;224(04):252-254. DOI: 10.1055/s-2007-962849.

23. Heijl A, Patella VM, Chong LX, et al. A New SITA Perimetric Threshold Testing Algorithm: Construction and a Multicenter Clinical Study. Am J Ophthalmol 2019;198:154-165. DOI: 10.1016/j.ajo.2018.10.010.

24. Bujang MA, Adnan TH. Requirements for minimum sample size for sensitivity and specificity analysis. J. Clin Diagn Res 2016;10(10):YE01. DOI: $10.7860 / J C D R / 2016 / 18129.8744$.

25. Sponsel WE, Ritch R, Stamper R, et al. Prevent Blindness America visual field screening study. Am J Ophthalmol 1995;120(6):699-708. DOI: 10.1016/s0002-9394(14)72723-0.

26. Phu J, Khuu SK, Yapp M, et al. The value of visual field testing in the era of advanced imaging: clinical and psychophysical perspectives. Clin Exp Optom 2017;100(4):313-332. DOI: 10.1111/cxo.12551.

27. Bengtsson $B$, Heijl A. False-negative responses in glaucoma perimetry: indicators of patient performance or test reliability? Invest Ophthalmol Vis Sci 2000;41(8):2201-2204. DOI: 10.1016/S00029394(00)00758-3.

28. Hajian-Tilaki K. Receiver operating characteristic (ROC) curve analysis for medical diagnostic test evaluation. Caspian J Intern Med 2013;4(2):627.

29. Foster PJ, Buhrmann R, Quigley HA, et al. The definition and classification of glaucoma in prevalence surveys. $\mathrm{Br} J$ Ophthalmol 2002;86(2):238-242. DOI: 10.1136/bjo.86.2.238.

30. Šimundić A-M. Measures of diagnostic accuracy: basic definitions. EJIFCC 2009;19(4):203.

31. Mukaka MM. A guide to appropriate use of correlation coefficient in medical research. Malawi Med J 2012;24(3):69-71.

32. Lowry EA, Hou J, Hennein L, et al. Comparison of peristat online perimetry with the Humphrey perimetry in a clinic-based setting. TransI Vis Sci Technol 2016;5(4):4-4. DOI: 10.1167/tvst.5.4.4.

33. Brusini $P$, Salvetat $M L$, Parisi $L$, et al. Probing glaucoma visual damage by rarebit perimetry. Br J Ophthalmol 2005;89(2):180-184. DOI: 10.1136/bjo.2003.041178.

34. Willis BH. Spectrum bias-why clinicians need to be cautious when applying diagnostic test studies. Fam Pract 2008;25(5):390-396. DOI: 10.1093/fampra/cmn051.

35. Robin TA, Müller A, Rait J, et al. Performance of community-based glaucoma screening using Frequency Doubling Technology and Heidelberg Retinal Tomography. Ophthalmic Epidemiol 2005;12(3):167-178. DOI: 10.1080/09286580590969716.

36. Etikan I, Musa SA, Alkassim RS. Comparison of convenience sampling and purposive sampling. Am JTheoret Appl Statist 2016;5(1):1-4. DOI: 10.11648/j.ajtas.20160501.11. 